\title{
Theoretical and experimental researches on the implementation of smart location
}

\author{
Mariana Panaitescu ${ }^{1,},{ }^{*}$ Fanel-Viorel Panaitescut ${ }^{1}$, Marius-Valentin Dumitrescu ${ }^{2}$, and \\ Valeriu-Nicolaie Panaitescu ${ }^{2}$ \\ ${ }^{1}$ Electromechanical Faculty, Engineering Sciences in mechanics and Environment Department, \\ 900663 Mircea cel Batran Street, Romania \\ 2 Doctoral School of Energy Engineering, POLITEHNICA University, Bucharest, Romania
}

\begin{abstract}
The present paper considers the presentation of practical solutions for the realization of smart location, materialized through research on solutions for monitoring parameters and solutions for these locations.In this paper we present: description of the concept of smart location, structures and architectures, realization of a pilot installation of smart location and data aquisition. For these researches was used to monitor electro-mechanical radiation of its own design. We obtained the following results: realization and design of the elements of the housing components with the $123 \mathrm{D}$ Design program, processing with the Cura program, obtaining g-code files for transfer to 3D printing, monitors in real time and detects errors or deficiencies in location. The main objective of this paper is to propose a model of technical solution for an smart location, which can be applicable in the maritime field, respectively to ships in the engine room or other location onboard.
\end{abstract}

\section{Introduction about smart location}

The present paper considers the presentation of practical solutions for the realization of smart locations, materialized through research on solutions for monitoring parameters and solutions for these locations.

Now that the construction of "Intelligent Locations compatible with IoT" is in full swing, it was considered that a research and understanding of systems and how to operate, followed by a realization at the level of the model would be auspicious [1]. "Intelligent Locations is an Internet of Things (IoT) ..... that provides.... with data insights gained through advanced monitoring of assets, people and processes to solve persistent problems of inefficiency, affordability and accessibility." [2].

By definition: "An intelligent locations represents a fusion of technologies, projectors and energy systems. A smart home has primary and secondary data buses that connect highlevel controllers with lower-level controllers, input / output devices, and the user interface

* Corresponding author: panaitescumariana1@gmail.com 
(Figure 1) [2]. Primary and secondary data buses can be CAN, BACnet, fiber optic, ethernet, ARCNET, RS-232, RS-485 or wireless ".[1]

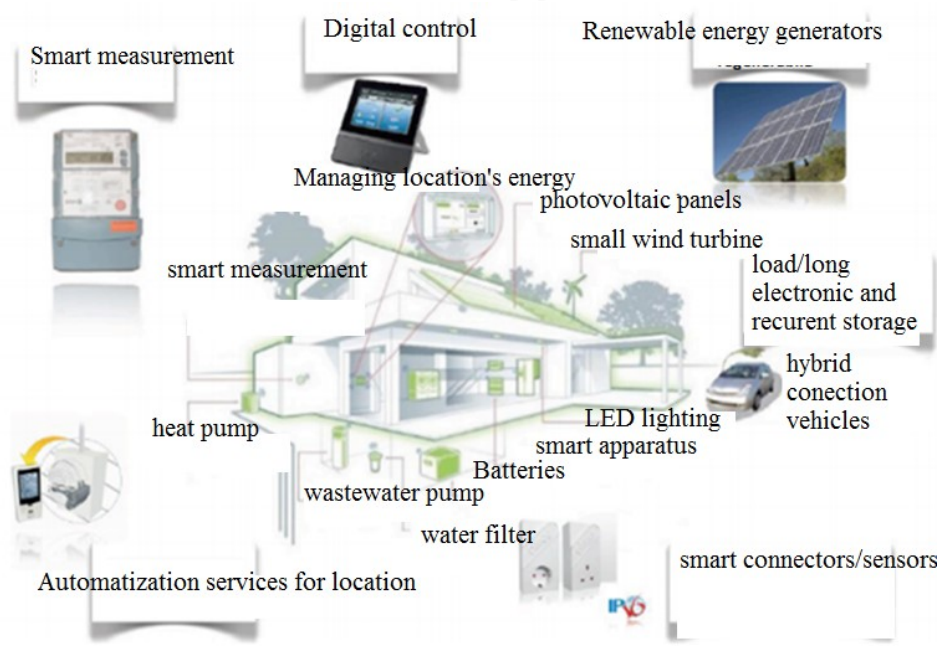

Fig. 1. Intelligent location.

Now we can summarize the concept of "Smart Location" as the fusion of technology, design and energy systems (Figure 2). They are well managed, have well-integrated physical and digital infrastructure that provides optimal employment services in a reliable, cost-effective and sustainable way.

The smart location helps its users to improve their lifestyle, by improving the technical performance of the location, reducing consumption, implicitly expenses, increasing reliability, optimizing space and last but not least, minimizing the environmental impact of locations. Smart location can be :" Smart Cities where sensors can offer us services like Smart Parking - to find free parking spots in the streets- or managing the intensity of the luminosity in street lights to save energy"[3]. This concept means "specific sensors and wireless technologies for each application" [3].

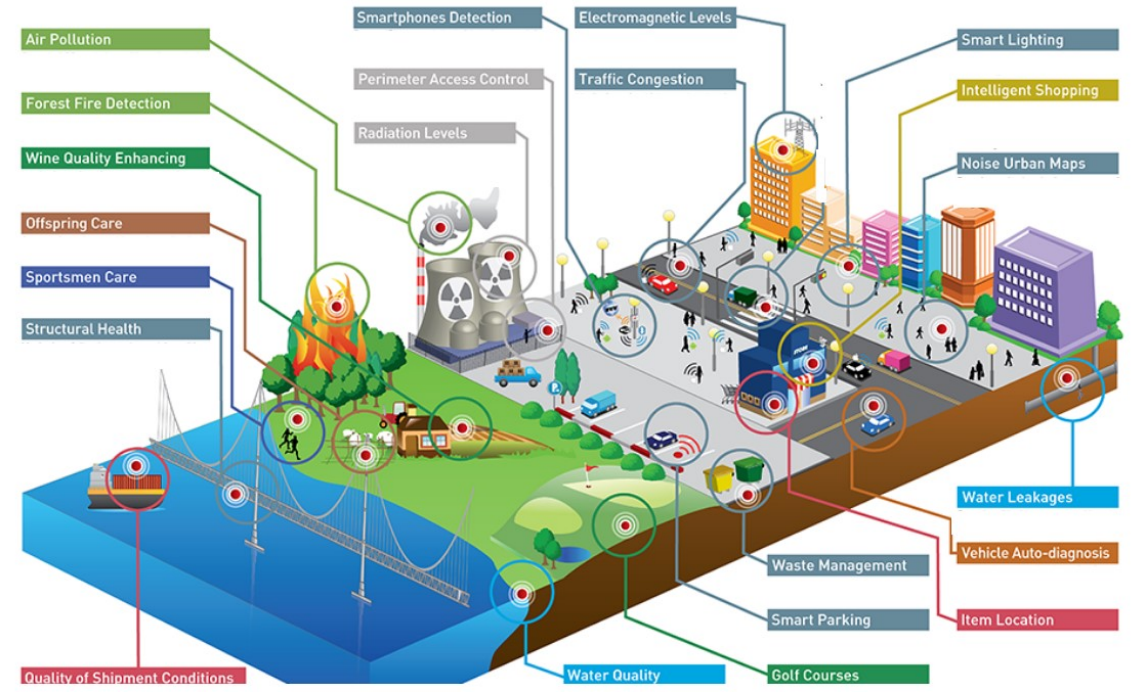

Fig. 2. Smart location. 
The main objective of this paper is to propose a model of technical solution for an smart location, which can be applicable in the maritime field, respectively to ships in the engine room or other location onboard.

A smart location:

- $\quad$ provides data on the performance of building systems;

- monitors in real time and detects errors or deficiencies in construction systems;

- $\quad$ reports in real time and uses operations management, energy and tenant comfort;

- includes tools, technologies, resources and systems to contribute to the conservation of energy and environmental sustainability.

"(IoT) is a "system of systems" in which people, machines, devices, sensors and businesses connect and interact"[4].

In maritime field, onboard, in the engine room or other location, the smart location is workplace, where "as devices continue to connect employees, they are creating much more than an office - they are creating collective intelligence. Applications abound in workplace safety, employee productivity, asset management and building comfort'[4].

A smart location onboard means a part of smart ship which consists of four main elements (Figure 3)[5]:navigation, guidance, physical ship and controls.

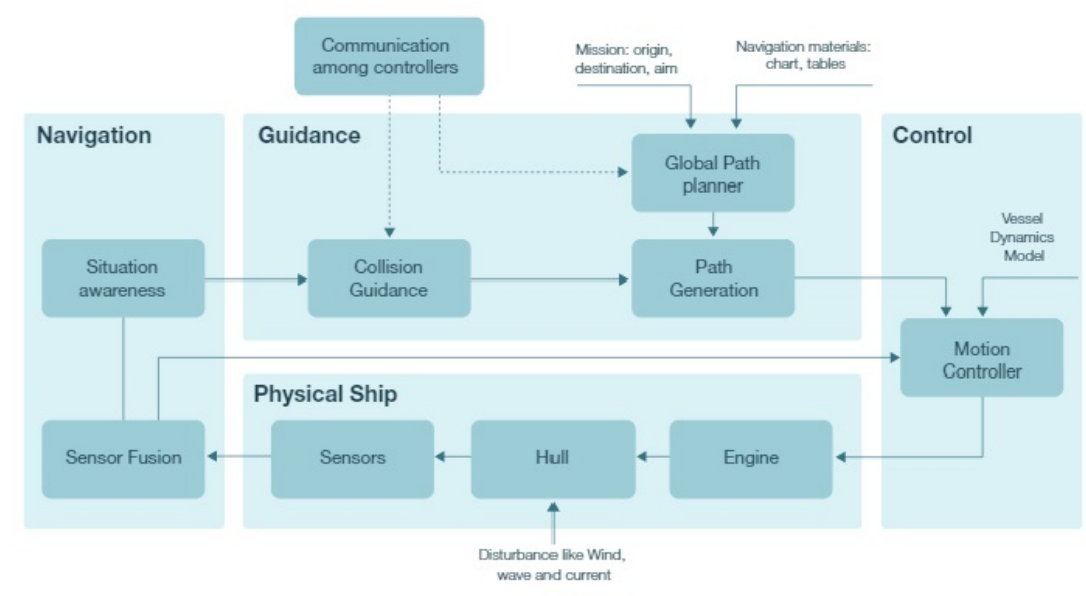

Fig. 3. Smart ship.

In this paper we describe a part of physical ship- engine, a smart pilot installation of this location and data aquisition.

\subsection{Architectures and structure}

\subsubsection{Architectures}

The architecture of a sensor network is dependent on the application for which it is used and in which it is integrated. Temperature and humidity sensors, a HID interface (Human Interface Devices)- Display were used and a logic board Esp 32 (Figure 4). 

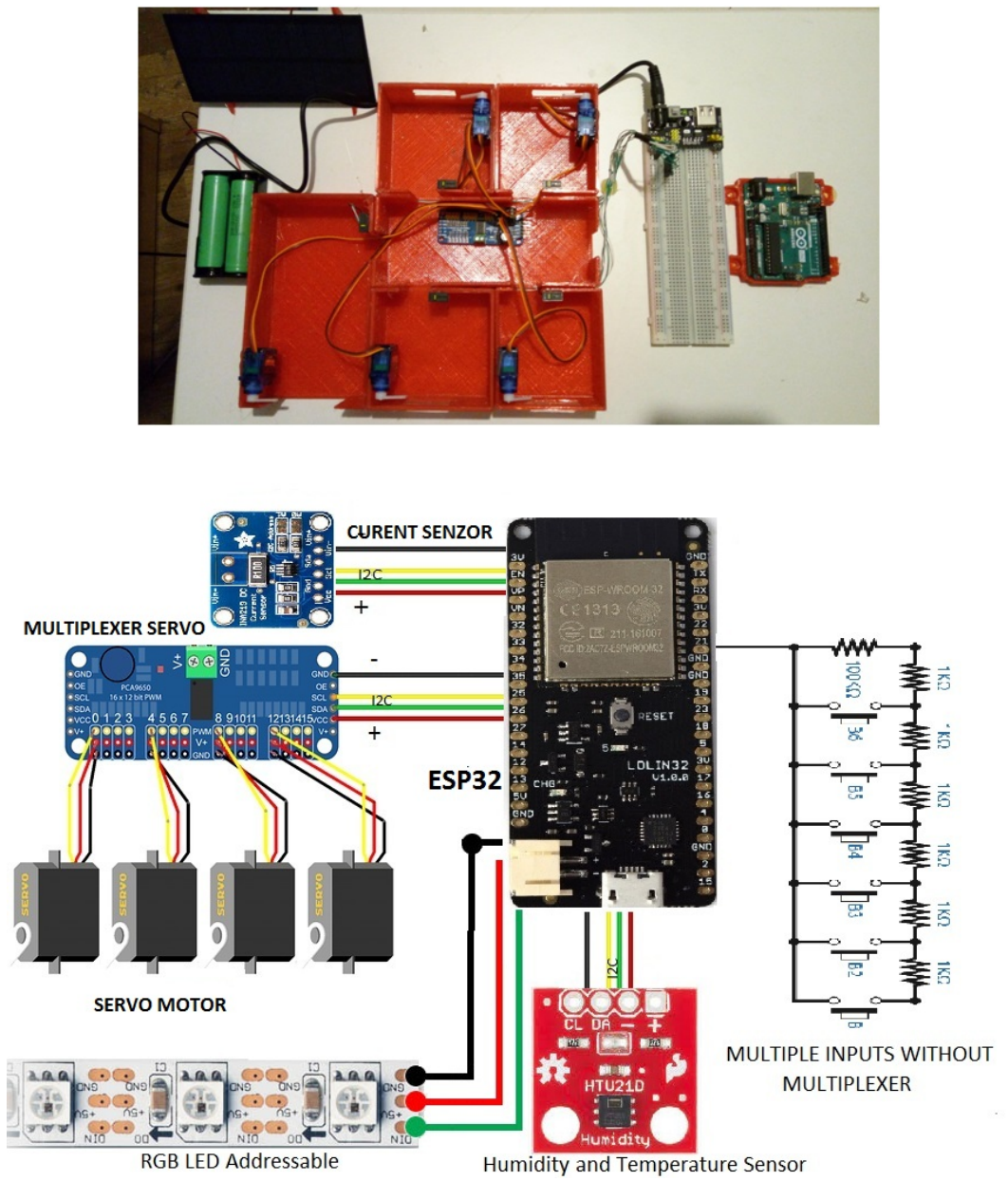

Fig. 4. The architecture of a sensor network.

\subsubsection{Structure}

The network of automation of an intelligent fall is an interconnection of hardwares and centralized software that monitors and controls the industry, the environment and the environment (Figure 5) [5], [6].

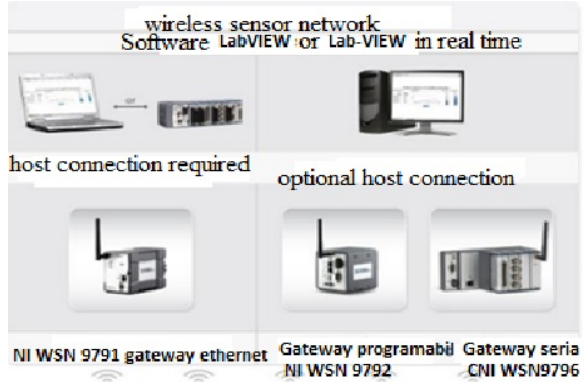

Fig. 5. Wireless sensor network. 


\subsection{Smart location pilot structure}

The location structure: four rooms, a hall and a warehouse, equipped with "gadgets" for transformation into a Smart Location [7], [8].

The gadgets that have been used for data acquisition are: various sensors and switches Normally Open [9].

For data main line we will use the $\mathrm{I}_{2} \mathrm{C}$ protocol, similar to the CAN and RS232 protocol used in professional systems [10].

For execution we will use addressable LEDs and servomotors, and for interface we will use OLED display [11].

The logic board has an ESP32 processor, compatible with Arduino development boards and the Arduino IDE program [16]. For programming the ESP32 module, the Arduino IDE programming environment was used, helped by the libraries related to sensors and multiplexers. The possibility of internet connection and the existence of the internal memory of the ESP32 module facilitated the creation and implementation of a web page accessible online by accessing the data acquired by the sensors [10]. ESP32 is a series of low-cost, low-power system on a chip microcontrollers with integrated Wi-Fi and dual-mode Bluetooth. It also has the possibility to connect to the internet, in order to transform the compatible IoT project (Figure 6) [11].

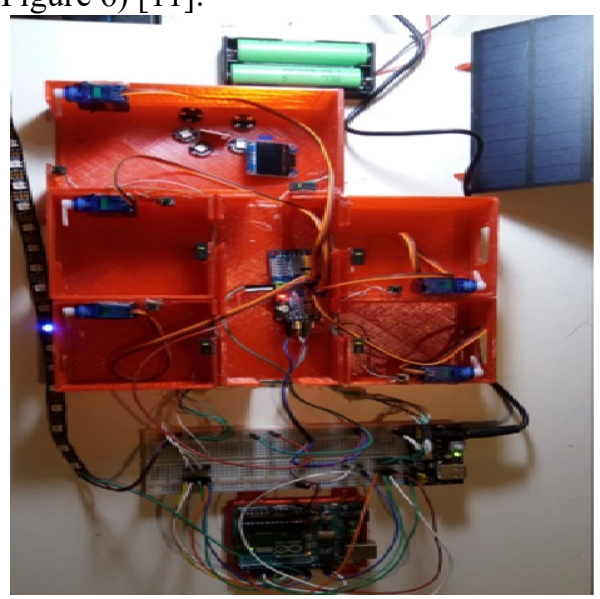

Fig. 6. Pilot smart structure.

\section{Realization and design of the elements of the housing components}

\subsection{Design program}

The components of the pilot installation were made with the 123D Design Program (Figure 7) [12]." Autodesk 123D was a suite of hobbyist CAD and 3D modelling tools created by Autodesk."123D Design Program is a simplified program to create 3D models. 
Fig. 7. The hall design.

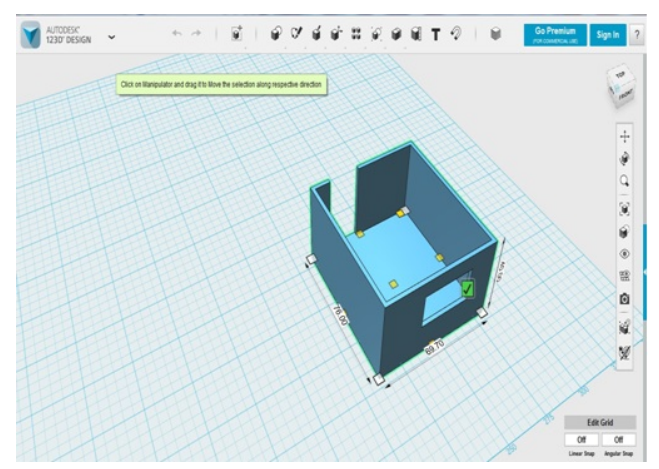

The created elements were exported in Stl files and then processed with the Cura program to obtain g-code files." Cura is an open source slicing application for 3D printers"[13]. The latter were transferred to the 3D printer and processed for the final shape. "Ultimaker Cura works by slicing the user's model file into layers and generating a printer-specific g-code. Once finished, the g-code can be sent to the printer for the manufacture of the physical object."'[14], [15]

\subsection{Engine Room design}

The design of the engine rooms was done as follows:

- designing the hall (Figure 7) and the whole smart location (Figure 8);

- design of storage (Figure 9);

- processing with the Cura program, obtaining Gcode files for transfer to 3D printing (Figure 10, Figure 11, Figure 12, Figure 13);

- File transfer and 3D printing (Figure 14).

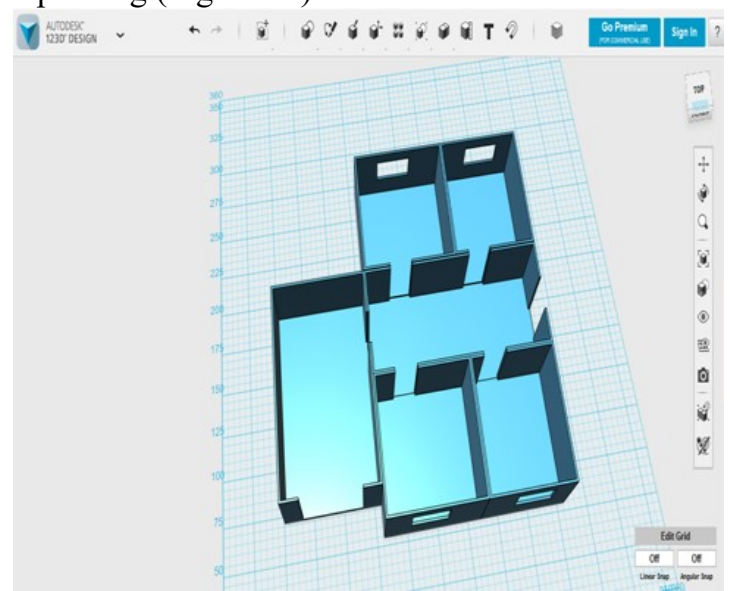

Fig. 8. The whole smart location design. 


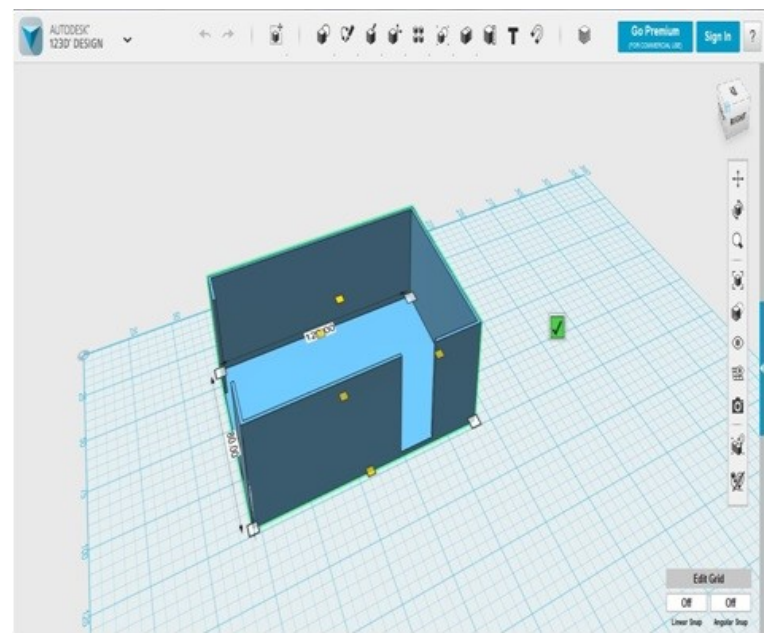

Fig. 9. The store design.

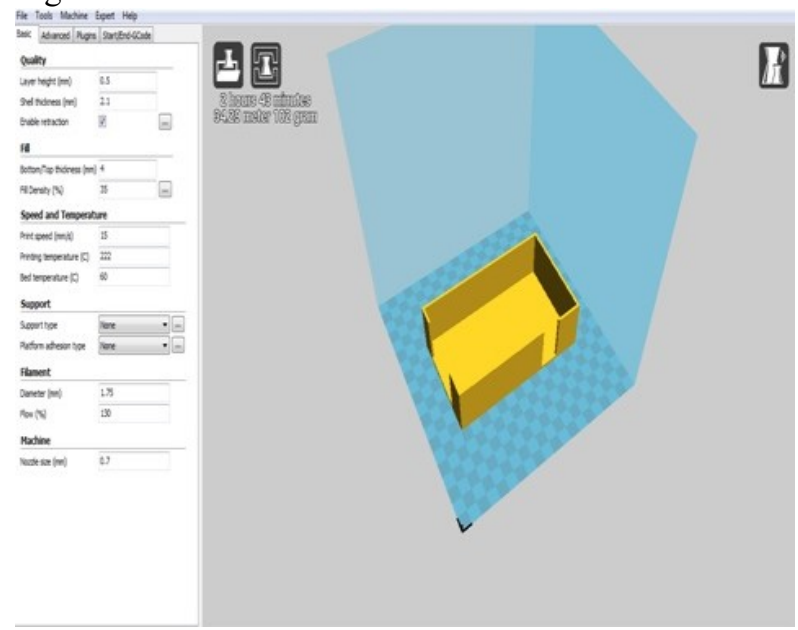

Fig.10. The hall- processing with the Cura program.

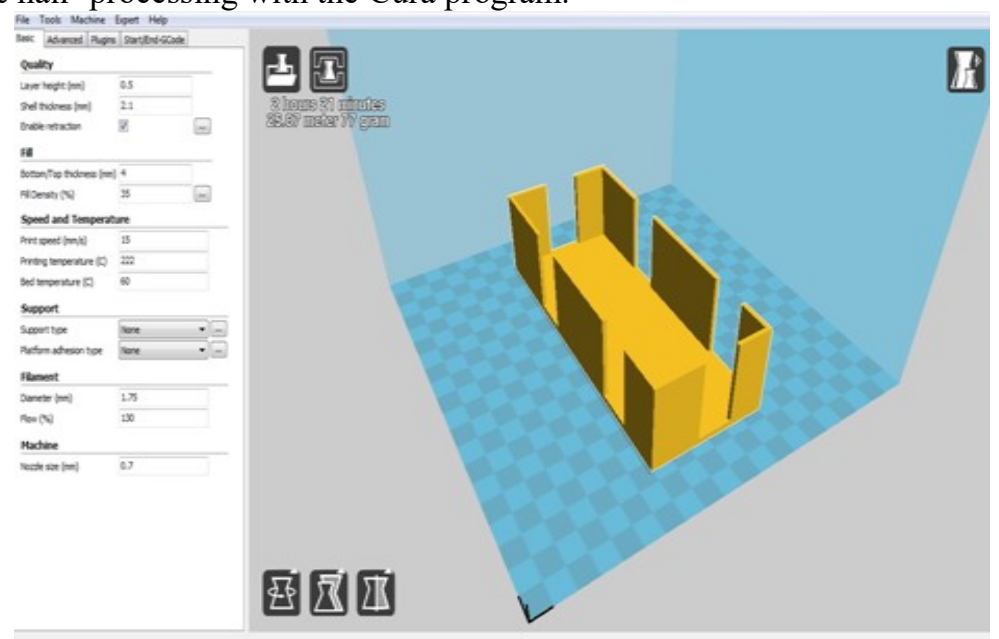

Fig.11. The rooms- processing with the Cura program. 


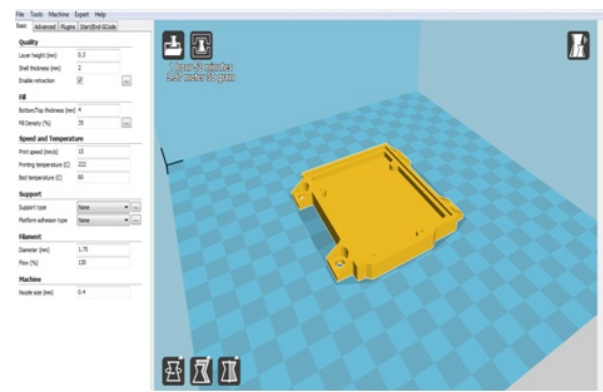

Fig.12. The materboard- processing with the Cura program.

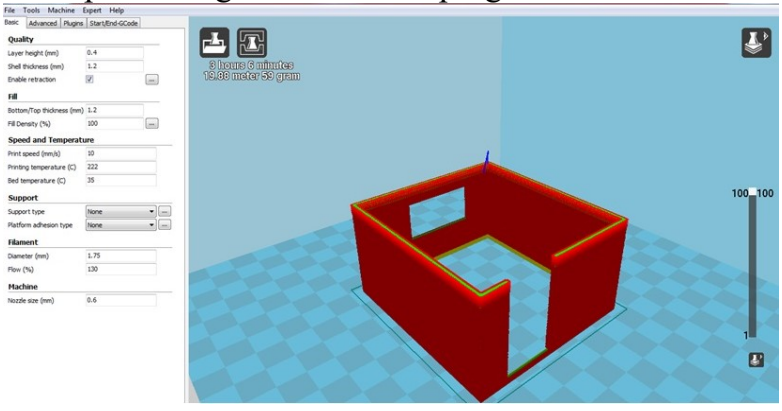

Fig.13. The storage room- processing with the Cura program.

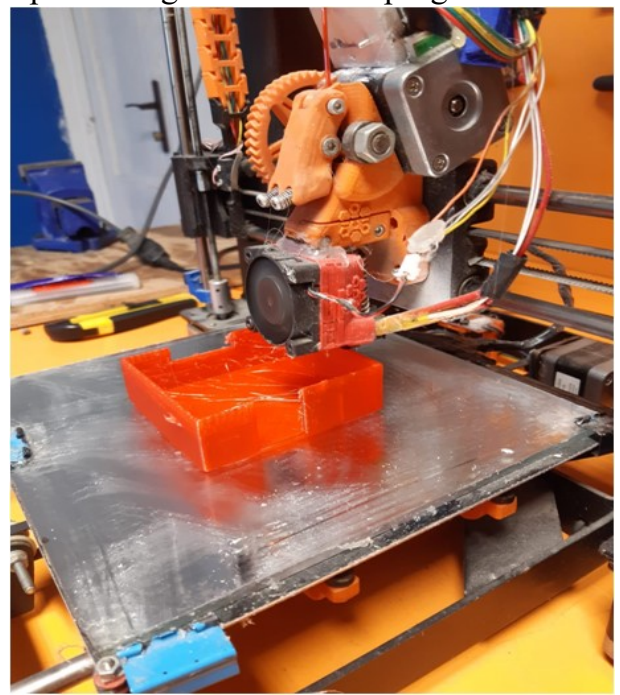

Fig.14. File transfer and 3D printing.

\subsection{Assembling the location}

\section{Motherboard:}

- on a melamine chipboard support.

- Actuation, closing and opening of blinds and storage room doors - with actuators and control microcontacts - actuation and ignition of interior lights with a control such as push on / push off circuit.

Positioning of electronic elements: 
The board is acting as a connection panel, interconnecting the respective electronic elements:

Energy sources: photovoltaic panel and network.

Control elements: NO type buttons

Execution elements: Servomotors, through tca9548a i2c multiplexer, LED lighting.

Sensors: Humidity and temperature sensor HTU21D, Current-Sensor INA219

HID (Human Interface Devices): Display

Logic board: Esp 32 or Esp8266.

\subsection{Installation of connections and actuators}

In order to obtain the final structure of the pilot installation, the connections and actuators are mounted (Figure 15, Figure 16).

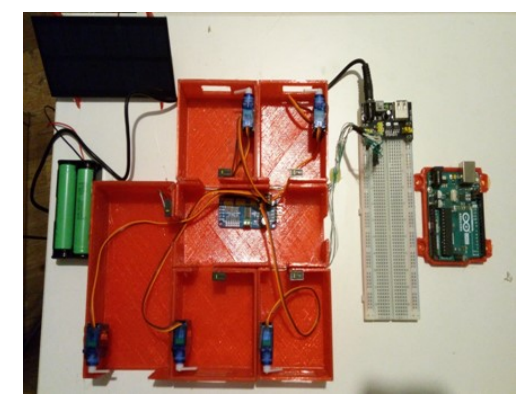

Fig.15. Installation of connections.

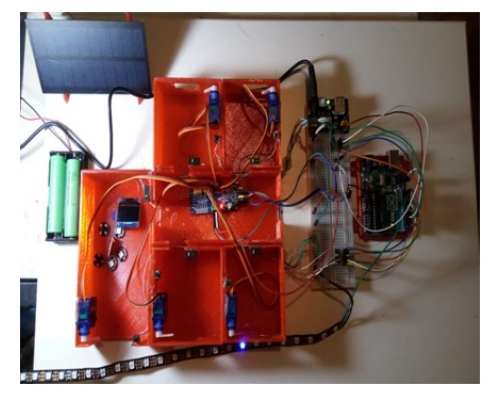

Fig.16. Installation of actuators.

\section{Smart location assembly operation}

By pressing the NO (round) buttons, an electrical signal is transmitted to the logic board which, through the I2C bus, transmits to the controller for the actuators the opening or closing of the blinds or the garage door.

The actuators can be pre-programmed at which angle to open or close.

By pressing the NO (rectangular) buttons, an electrical signal is transmitted to the logic board, which transmits the on or off LEDs to the addressable LEDs. The color and intensity 
of the LED light can be preset from the software. They can ignite according to a predefined code for issuing warnings (burglary, fire, etc.).

Also, their software can run in anti-burglary system that mimics the presence of tenants in the house, when they are away from home for a longer period.

The sensors connected to the I2C bus transmit data collected from the home to the logic board, which can be accessed by the owners via the Internet. HTU21D is a digital humidity sensor with temperature output [17]. This sensor provides calibrated, linearized signals in digital, $\mathrm{I}^{2} \mathrm{C}$ format. HTU21D digital humidity sensors are dedicated humidity and temperature plug. The INA219 Current-Sensor is an I2C interface based zero drift and bidirectional current/power monitoring module [18]. It can sense shunt voltage, current, and power at the same time and submit the data via $\mathrm{I} 2 \mathrm{C}$ protocol.

\section{Data from the trial period of the project on the mobile phone}

Through the internet you can order the start or stop of the different systems that equip the home.

In the realized project we used as elements that simulate actuation, direct current servo motors. Besides the simple on / off functions they can also execute angular movements necessary in partial openings, ventilation systems, positioning systems and others.

Micro Servo Motor SG90, used in this project, is a tiny and lightweight server motor with high output power. Servo can rotate approximately 180 degrees ,90 in each direction.

Starting, stopping and setting the home heating, watering systems, animal feed, space ventilation, ambient light, etc. For our smart location we have collected daily and weekly data (Table 1, Figure 17, Figure 18).

Table 1. Daily and weekly variation on temperature and umidity.

\begin{tabular}{|c|c|c|}
\hline Day/hour & ${\text { Temperature }\left[{ }^{\mathbf{0}} \mathbf{C} \text { ] }\right.}^{\text {Umidity [\%] }}$ \\
\hline $\begin{array}{c}5 / 15 / 2021 \\
9: 21: 00 \mathrm{PM}\end{array}$ & 22 & 39.1 \\
\hline $\begin{array}{c}5 / 15 / 2021 \\
8: 21: 00 \mathrm{PM}\end{array}$ & 22 & 39.1 \\
\hline $\begin{array}{c}5 / 15 / 2021 \\
6: 51: 00 \mathrm{PM}\end{array}$ & 22.4 & 38.6 \\
\hline $\begin{array}{c}5 / 15 / 2021 \\
6: 01: 00 \mathrm{PM}\end{array}$ & 22.3 & 39.1 \\
\hline $\begin{array}{c}5 / 15 / 2021 \\
2: 41: 00 \mathrm{PM}\end{array}$ & 22 & 39 \\
\hline $\begin{array}{c}5 / 15 / 2021 \\
1: 21: 00 \mathrm{PM}\end{array}$ & 22 & 39.1 \\
\hline $\begin{array}{c}5 / 15 / 2021 \\
12: 51: 00 \mathrm{AM}\end{array}$ & 21.5 & 40.6 \\
\hline $\begin{array}{c}5 / 15 / 2021 \\
12: 11: 00 \mathrm{PM}\end{array}$ & 21.9 & 39.6 \\
\hline
\end{tabular}




\begin{tabular}{|c|c|c|}
\hline $5 / 15 / 2021$ & 21.7 & 40.1 \\
$9: 21: 00 \mathrm{AM}$ & 21.5 & 40.6 \\
\hline $5 / 15 / 2021$ & & \\
$3: 41: 00 \mathrm{AM}$ & & \\
\hline
\end{tabular}

\section{Temperature [0C]}

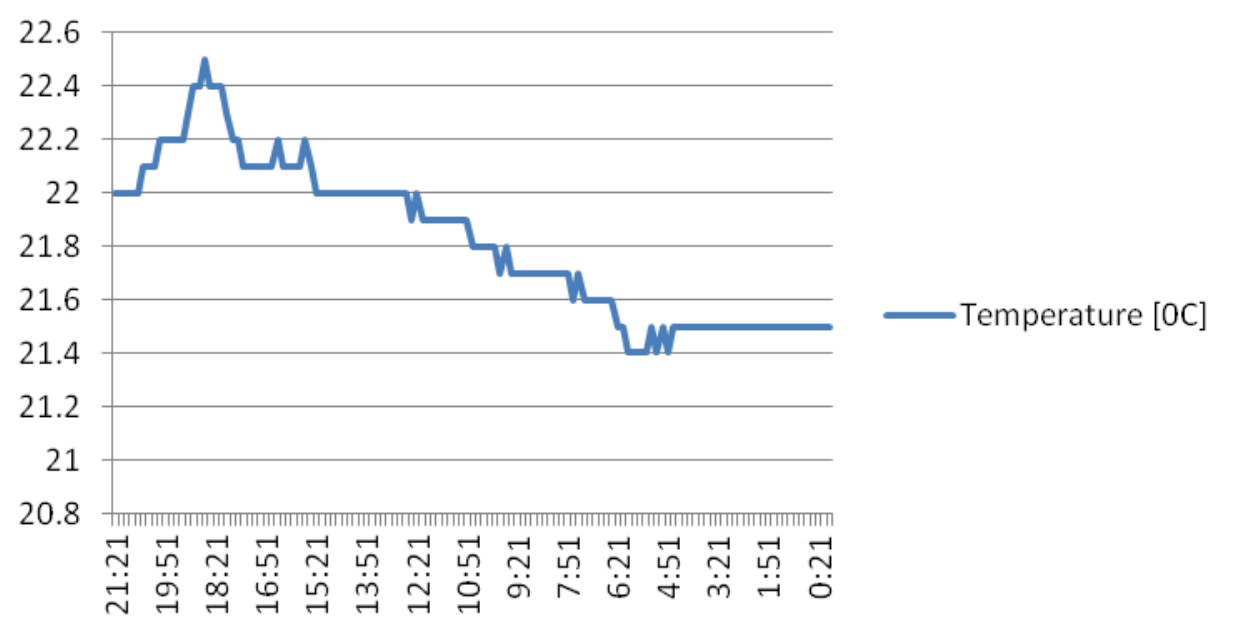

Fig.17. Daily variation on temperature.

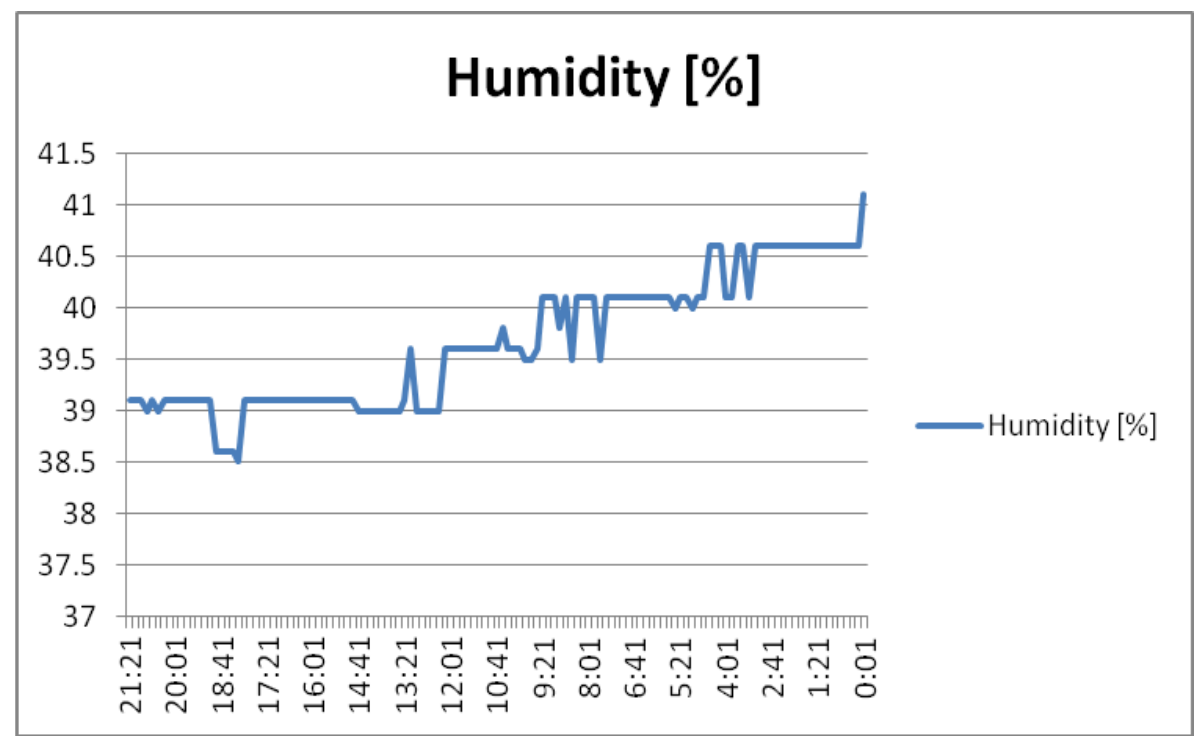

Fig.17. Daily variation on humidity. 
The location can be provided with a connection to a photovoltaic system, consisting of a solar panel, buck boost inverter and battery (battery), which provides electricity on sunny days, thus contributing to the application of the concept of environmental sustainability.

Other data we can find on http://5.14.172.250.

\section{Conclusions}

The purpose of this project, that of finding practical solutions to carry out "smart location" projects without having to invest large sums of money and with logistical resources at the disposal of many entities, has been achieved. Following the experience gained in carrying out the exercise, we can say that the technical solution model created by us can be easily adapted and replicated for other environments. In the naval field, the main objective is to monitor the environment in the engine room or other places of interest on board ships.

The cost reduction was due to the use of 123D Design, Cura and Arduino IDE programs with freeware license. Also, the implementation of the I2C communication protocol, which does not require the payment of a license, is also a cost-reducing solution. This communication protocol allows us to attach a total of 128 acquisition or execution equipment.

The use of cheap, pre-calibrated micro-sensors developed by renowned companies at the urging of the European Community able to offer I2C compatibility was another correct choice in this approach.

The chosen technical solution offered the user access to a wide range of essential data from the monitored environments such as:

- information about energy sources with monitoring of essential parameters, voltage, current.

- information about the parameters of air quality, temperature, humidity, atmospheric pressure,

- the system can issue alarms, both locally and remotely, when the preset values for the monitored elements are exceeded.

The motivation for choosing this type of work for this project was to ultimately serve as a teaching material for our students bringing ease in understanding the operation of IoT systems. We also leave the possibility of their involvement and further development of such a project.

\section{References}

1. Daniel Wilson. Smart House-Your So-Called Sci-Fi Life", Popular Mechanics Technology News, Mai (2007)

2. https://www.prnewswire.com/news-releases/intelligent-locations-and-sas-collaborateto-support-healthcare-organizations-iot-and-contact-tracing-efforts-301114734.html

3. https://www.libelium.com/libeliumworld/top-50-iot-sensor-applications-ranking/

4. https://isg-one.com/articles/iot-and-the-intelligent-workplace

5. Mariana Panaitescu, Marius Valentin Dumitrescu, Fanel-Viorel Panaitescu. Indoor air quality monitoring universal device, Proc.of the 8th International Conference on 
Modern Manufacturing Technologies in Industrial Engineer ISSN 2286-4369, Iasi, Romania ( June 23-27, 2020).

6. Viegi Giulio. Indoor air pollution and airway disease, Int.J.Tuberc.Lung Disease, 8, 1401 (2004)

7. Dumitrescu Marius-Valentin, Studies on techniques and technologies, 2-86, Bucharest, Romania (2019)

8. Panaitescu Mariana, Panaitescu Fanel-Viorel, Dumitrescu Marius-Valentin, Panaitescu Valeriu Nicolae, The Impact of Quality Air in The Engine Room on the Crew, Int.J.of Marine Navigation and Safety of Sea Transportation V1, Isue 3.2, 409-414 (2019)

9. Vasile BAHRIN, Câmpul electromagnetic de radiație generat de echipamentele electrice, Electrician J. 06,6-12 (2014)

10. Espressif System ESP32, Technical Reference Manual V4.1, 285-286 (2019)

11. Leszczynski D., Joenv S., Reivinen J, and Kuokka R., Non-thermal activation of the hsp27/p38MAPK stress pathway by mobile phone radiation in human endothelial cells: Molecular mechanism for cancer and blood-brain barrier-related effects, Differentiation J., 70, 120 - 129 (2002)

12. https://en.wikipedia.org/wiki/Autodesk_123D.

13. "Cura home page". Ultimaker. Ultimaker. Retrieved 31 December 2016

14. https://all3dp.com/cura-2-3-update-new-features-major-improvements-3d-slicer.

15. https://3dprint.com/211217/ultimaker-engineering-materials.

16. https://www.arduino.cc/en/software.

17. https://cdn-shop.adafruit.com/datasheets/1899_HTU21D.pdf.

18. https://www.ti.com/product/INA219.

\section{Acknowledgments}

Authors gratefully acknowledge to this material support path received under Project PN-IIIP1-1.2-PCCDI-2017-0404/ 31PCCDI / 2018, Holistic on the Impact of Renewable Energy Sources on Environment and Climate-HORESEC. 